\title{
Assessment of Knowledge and Attitude of Pediatricians and General Dentists toward Infant Oral Health Care, Jaipur
}

\author{
Shavan K Yadav ${ }^{1}$, Anant G Nigam² ${ }^{2}$, Renuka P Chinchalkar ${ }^{3}$, Mili R Meghpara ${ }^{4}$, Pooja Yadav ${ }^{5}$
}

\begin{abstract}
Introduction: Oral health is the foundation upon which dental health must be built. Pediatricians examine infants several times during their 1st and 2nd years of life. Parents usually seek pediatricians' advice in securing their child's normal growth and development. The aim of this study was to assess the knowledge and attitude regarding infant oral health in pediatricians and general dentist and to make aware about the preventive oral health care $(\mathrm{OHC})$ needs for the infants to their parents.

Materials and methods: A total of 100 pediatricians and 100 general dentists who participated in the study were randomly selected. Objective questionnaires related to infant $\mathrm{OHC}$ were distributed among the pediatricians and general dentists and asked to fill the pro forma. They were requested to fill an objective questionnaire without providing any oral health information.

Results and conclusion: The results were statistically analyzed and it was concluded that there was a lack of knowledge among pediatricians as compared to the general dentist but overall both lacked the current concepts of pediatric dentistry.

Keywords: Attitude, General dentists, Infant oral health care, Knowledge, Pediatricians.

Journal of Mahatma Gandhi University of Medical Sciences \& Technology (2020): 10.5005/jp-journals-10057-0138
\end{abstract}

\section{INTRODUCTION}

Children and young adults often develop deep carious lesions due to poor oral hygiene and maintenance. These carious lesions if not treated early lead to deeper carious lesions eventually involving the pulp. ${ }^{1}$ One of the significant challenges posed by the dental fraternity is the maintenance of a population with good oral health. The dental disorder is not just a minor disease of the gums and teeth, but a disease of the body that happens to commence in the oral cavity. $^{2}$

Working with children presents sole challenges to a dentist, especially the endodontic treatment in the primary teeth. The main problem with pulpally involved primary tooth is the diagnosis. Even after diagnosis, the correct treatment protocol for pediatric endodontics, i.e., instrumentation, working length determination, and obturation, has to be met, which are quite a challenge in a primary tooth, so that the primary tooth can be saved without any further complications. Most of the time, the treatment done remains incomplete either due to lack of knowledge of the dentist or due to non-cooperation on the part of the child or his/her parents. ${ }^{3}$ Many dental practitioners generally prefer to extract the primary pulpally involved tooth because of these challenges. Knowing the importance of primary teeth in maintaining the arch length and prevention of malocclusion we have to keep the primary teeth free of infection. ${ }^{4}$

Infant oral health is the foundation upon which dental health must be built. It is recommended that the first dental visit should occur within 6 months and no later than 12 months of age. The American Academy of Pediatrics previously recommended the first dental visit by age 3 but changed the guideline in 2003 to establish a dental home by age 1 for children with caries risk. ${ }^{5}$

Pediatricians examine infants several times during their 1st and 2nd years of life. Parents usually seek pediatricians' advice in securing their child s normal growth and development. If proper counsel regarding the child's development and eruption of teeth
${ }^{1-5}$ Department of Pedodontics and Preventive Dentistry, Mahatma Gandhi Dental College and Hospital, Jaipur, Rajasthan, India

Corresponding Author: Shavan K Yadav, Department of Pedodontics and Preventive Dentistry, Mahatma Gandhi Dental College and Hospital, Jaipur, Rajasthan, India, Phone: +91 8652123527, e-mail: shavany092@gmail.com

How to cite this article: Yadav SK, Nigam AG, Chinchalkar RP, et al. Assessment of Knowledge and Attitude of Pediatricians and General Dentists toward Infant Oral Health Care, Jaipur. J Mahatma Gandhi Univ Med Sci Tech 2020;5(2):69-71.

Source of support: Nil

Conflict of interest: None

as well as guidance for the prevention of various oral diseases is rendered at this age, better oral health can be attained for these children. ${ }^{6}$

The need of the study is to assess the knowledge and attitude regarding infant oral health in pediatricians and general dentists and to make aware about the preventive oral healthcare $(\mathrm{OHC})$ needs for the infants to their parents.

\section{Materials and Methods}

This study was done in Jaipur, with a total of 100 pediatricians who had a Postgraduate Diploma (DCH) or a Master's Degree (MD) or both, DNB and other degrees in pediatrics, and 100 general dentists participated in the study. For the study purpose, objective questionnaires were distributed among the pediatricians and general dentists and asked to fill the pro forma. Earlier appointments and the schedule for the study were obtained from the respective pediatricians and general dentists. The questionnaire included was related to infant $\mathrm{OHC}$. They were requested to fill an objective questionnaire without providing any oral health information. The questionnaire pro forma was self-prepared. Validation of the questionnaire was done (Table 1). 
Table 1: Questionnaire pro forma

1. Profession

a. General dentist b. Pediatrician

2. When does the 1st primary tooth erupt?
a. 6 to 8 months
b. 9 to 11 months
c. 12 to 18 months
d. After 18 months

3. Which is more important in causing dental caries?
a. Amount of sugar intake
b. Frequency of sugar intake
c. I don't know

4. Are there any differences between breast milk and bottle feeding regarding their effect on dentition?
a. Yes b. No
c. I don't know

5. When should a child stop thumb-sucking habits?
a. After the primary dentition has completed
b. Before the eruption of the permanent incisor
c. After the eruption of the permanent incisors
d. No specific age

6. At what age should a child have his first dental visits?
a. At 2 years
b. At 1 year
c. At 6 months
d. I don't know

7. Does a child free of caries need to visit a dentist?
a. Yes b. No

8. What is the amount of toothpaste that should be applied?
a. Full-length
b. Pea size
c. I don't know

9. At what age should the child start brushing his teeth?

a. After the eruption of primary molars ( 2 years)

b. After the eruption of a primary tooth ( 6 months -1 years)

c. After the eruption of permanent incisors

d. I don't know

10. Do you know what pit and fissure sealants are?
a. Yes
b. No

11. Do you routinely examine the oral cavity and dentition of your patient and assess dental and skeletal age?
a. Yes
b. No

12. Do you provide dietary counseling to your patients regarding cariogenic food?
a. Yes
b. No

13. Should gum pads be cleaned?
a. Yes
b. No

14. Do you think you can answer parent questions about oral health?
a. Yes
b. No
c. Maybe

15. Do you think it is important to prescribe fluoride supplements to children?
a. Yes
b. No
c. Maybe

16. Do you think swallowing fluoridated toothpaste is hazardous to children?
a. Yes
b. No

17. Do you think it is important to know the drinking water source of your patient?
a. Yes
b. No

Contd...

18. Do you think night feeding may affect the teeth?
a. Yes
b. No
c. Maybe

19. Do you think that an avulsed tooth can be re-implanted?
a. Yes
b. No
c. Maybe

20. Should counseling on the prevention of dental caries be a part of routine health child care?
a. Yes
b. No
c. Maybe

21. How important are the deciduous?
a. Extremely important
b. Important
c. Less important
d. Not important

\section{Results}

The study group comprised of total 100 pediatricians and 100 general dentists. The data obtained were analyzed by using SPSS (Statistical Package for the Social Sciences SPSS Inc., IBM, India) version 20.0 for windows. Mean and standard deviation (SD) were calculated for each clinical parameter. A table with the collected data was created in Excel and the data were statistically analyzed using the Chi-square test and non-parametric statistical tests. Significance for all statistical tests was predetermined at a $p \leq 0.05$.

The results were statistically analyzed and it was concluded that there was a lack of knowledge among pediatricians as compared to the general dentist but overall both lacked the current concepts of pediatric dentistry. The above table and figure depict that the comparison of mean knowledge score regarding $\mathrm{OHC}$ among dentist was more with a mean score of 17.23, whereas the mean knowledge score of a pediatrician was 13.11. Also, it was observed that the difference of knowledge was statistically significant with $p<0.05$ (Table 2 and Fig. 1).

\section{Discussion}

The current study focuses on the crucial oral preventive role of pediatricians who are considered the primary link between dentists and children. Therefore, this study has been planned to investigate the knowledge and attitude of pediatricians and general dentist in Jaipur, to ensure that maximum preventive and interceptive benefits can be delivered to children in the field of oral health.

It shows that pediatricians' dental knowledge is limited. Similar findings were reported by Lewis et al. who reported that only $9 \%$ of US pediatricians answered four knowledge questions correctly. On the other hand, regarding AAPD and AAP recommendations, the majority of the pediatricians in Jeddah (74.4\%) did not believe in the concept of early dental visits. The majority of pediatricians feel that they were not able to provide information related to oral health. ${ }^{7}$ Half of the pediatricians were not aware of the biannual dental visit that is recommended by AAPD. Sabbagh et al. concluded that only $47.7 \%$ of pediatricians instructed their patients to visit the dentist; however, they did not check out parents' compliance or appointment availability. They either referred them to general dentists $(39.7 \%)$, or pediatric dentists $(23.4 \%){ }^{8}$

In the study by Kumari et al. ${ }^{9}$, about $65.2 \%$ of the pediatricians recommended the 1 st dental visit in 6 months to 1 year as compared to $40 \%, 52.5 \%$ by Shivaprakash et al..$^{10}$ and only $2.7 \%$ of pediatricians 
Table 2: Association of oral health knowledge score between dentists and pediatricians

\begin{tabular}{llllll} 
& & \multicolumn{3}{c}{ Knowledge score } & \\
\cline { 2 - 5 } Oral health knowledge & $N$ & Mean & Un & Unaired t value & $p$ value \\
\hline Dentists & 100 & 17.23 & 3.3 & 6.26 & $<0.0001$ \\
Pediatricians & 100 & 13.11 & 5.7 & & \\
\hline
\end{tabular}

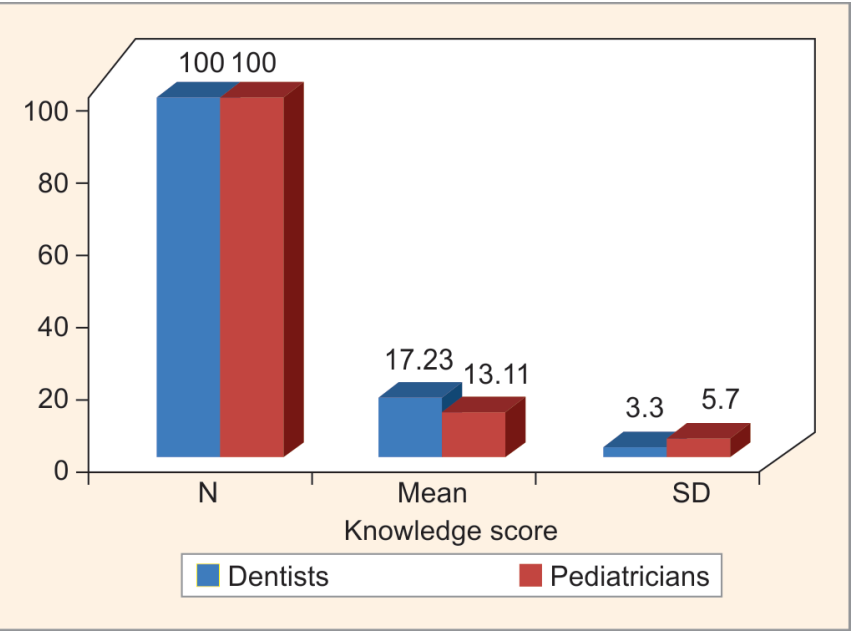

Fig. 1: Association of oral health knowledge score between dentists and pediatricians

and family physicians agreed in the study done by Preeti et al. ${ }^{11}$ As compared to the above-mentioned studies, all pediatricians recommended the 1st dental visit in 6 months.

In the present study with regards to dental knowledge, $67.3 \%$ of medical practitioners knew the importance of treating deciduous teeth and $60.7 \%$ of the respondents examined oral cavity routinely. However, Al-Hussyeen et al. reported that almost half of pediatricians did not routinely include dentition in their examinations. ${ }^{3}$

However, a focus on primary and secondary preventive $\mathrm{OHC}$ should occur for all children by both pediatricians and dentists. These two specialties need to work so that the oral health needs of all of their patients are met. Pediatricians establish early relationships with patients and their parents so they are having more faith in preventive advice starting from birth, while dentists are uniquely able to provide definitive treatment for dental disease. Therefore, both should have knowledge and awareness about current concepts of pediatric dentistry. ${ }^{12}$

\section{Conclusion}

Based on the findings of this survey, there was a lack of knowledge among pediatricians as compared to the general dentist but overall both lacked the current concepts of pediatric dentistry. There is a need for awareness about preventive $\mathrm{OHC}$ in infants to their parents. Further to increase knowledge among pediatricians and general dentists should be given importance.

\section{References}

1. McDonald RE, Avery DR, Dean JA, ed. Treatment of deep caries, vital pulp exposure and pulpless teeth. In: Dentistry for the Child and Adolescent. 8th ed., St. Louis: Mosby Elsevier; 2007. p. 396.

2. Patil A, Chavan S, Baghele ON. Awareness of oral health among medical practitioners in Sangamner city - a cross sectional survey. JIDA 2010;4(12):534-536.

3. Al-Hussyeen A, Al-Sadhan S, Al-Dhalaan R, et al. Pediatricians' knowledge and practices towards children's preventive oral health care in Saudi Arabia. Egyptian Dental J 2003;49:827-834.

4. Ahmed HM. Pulpectomy procedures in primary molar teeth. Eur J Gen Dent 2014;3(1):3-10. DOI: 10.4103/2278-9626.126201.

5. Brickhouse $T H$, Unkel JH, Kancitis I, et al. Infant oral health care: a survey of general dentists, pediatric dentists, and pediatricians in Virginia. Pediatr Dent 2008;30:147-153.

6. Shetty RM, Dixit UB. Paediatricians views on dental and oral health and treatment needs in children. Oral Health Prev Dent 2011;9:315322.

7. Sabbagh HJ, El-Kateb M, Al Nowaiser A, et al. Assessment of pediatricians dental knowledge, attitude and behavior in Jeddah, Saudi Arabia. J Clin Pediatr Dent 2011;35(4):371-376. DOI: 10.17796/ jcpd.35.4.8626721g8742102p.

8. Poornima P, Bajaj M, Nagaveni NB, et al. Evaluation of the knowledge, attitude and awareness in prevention of dental caries amongst paediatricians. Int J Commun Med Pub Health 2015;2(1):64-70. DOI: 10.5455/2394-6040.ijcmph20150213.

9. Kumari NR, Sheela S, Sarada PN. Knowledge and attitude on infant oral health among graduating medical students in Kerala. J Indian Soc Pedod Prev Dent 2006;24:173-176.

10. Shivaprakash PK, Elango I, Baweja DK, et al. The state of infant oral healthcare knowledge and awareness: disparity among parents and healthcare professionals. J Indian Soc Pedod Prev Dent 2009;27: 39-43.

11. Preeti P, Lawrence Herenia P, Harvey Bart J, et al. Early childhood caries and infant oral health: pediatricians and family physicians knowledge, practices, and training. Pediatr Clin Health 2006;11: 151-157.

12. Lewis CW, Boulter S, Keels MA, et al. Oral health and pediatricians: results of a national survey. Acad Pediatr 2009;9(6):457-461. DOI: 10.1016/j.acap.2009.09.016. 